\title{
Induction Immunotherapy Followed by Thoracic Radiation without Chemotherapy in Unresectable Stage III Non-Small Cell Lung Cancer: A Case Series
}

\author{
Srikiran Dasari ${ }^{1}$, Takefumi Komiya ${ }^{2 *}$
}

\begin{abstract}
Objective: Although concurrent chemoradiation has been the standard of care for unresectable stage III non-small cell lung cancer (NSCLC) due to increased survival and decreased disease progression, patients with poor performance status cannot tolerate chemotherapy toxicity well. Durvalumab, an immune checkpoint inhibitor targeting the programmed death receptor-1 (PD-1) / programmed death-ligand 1 (PD-L1) axis, demonstrated efficacy as maintenance therapy after definitive chemoradiation. However, the role of immunotherapy in those who cannot tolerate chemoradiation is unclear.Methods: This retrospective case series reports adult patients with PD-L1-expressing stage III NSCLC diagnosed at Parkview Cancer Institute from 2019-2021 and treated initially with pembrolizumab followed by sequential consolidation chest radiation (CXRT) without cytotoxic chemotherapy. Results: Four cases of stage IIIA squamous cell carcinoma were disease-controlled by this approach, with two partial and one complete response. One case of stage IIIC adenocarcinoma had progressive disease with brain metastasis prior to CXRT. Conclusion: This case series suggests that pembrolizumab with sequential CXRT may be beneficial for stage III NSCLC patients with high PD-L1 expression, but additional studies are needed to confirm this hypothesis.
\end{abstract}

Keywords: Non-small cell lung cancer- stage III- pembrolizumab- consolidation chest radiation- poor performance status

Asian Pac J Cancer Prev, 23 (1), 217-220

\section{Introduction}

Lung cancer is the number one cause of cancer-associated mortality in the United States, and the 5 -year overall survival rate for non-small cell lung cancer (NSCLC) has remained poor (Duma et al., 2019; American Cancer Society. 2021). For over a decade, the preferred treatment and standard of care for unresectable stage III NSCLC has been concurrent chemoradiation (Aupérin et al., 2010). Previous clinical trials demonstrated that concurrent chemoradiation was superior to sequential chemoradiation in terms of overall survival in patients with locally advanced NSCLC, albeit with increased acute esophageal toxicity (Aupérin et al., 2010; Mörth et al., 2014). However, this therapy is indicated primarily for patients with good performance status, and factors such as old age, co-morbidities, and poor performance status make it difficult for other patients to tolerate the toxicity of chemotherapy. For example, in a study of 31 NSCLC patients with poor performance status (PS $>2$ ) receiving platinum combination chemotherapy, those with a malnourished nutritional status according to the Glasgow Prognostic Score were unable to complete as many chemotherapy cycles as those in the well-nourished group and had poorer outcomes in terms of response rate, median progression-free survival (PFS), and median overall survival (Fujio et al., 2019).

Thus, immunotherapy, specifically immune checkpoint inhibitors with better tolerability, must be considered for this patient population as an alternative approach. Immune checkpoint inhibitors targeting the programmed death receptor-1 (PD-1) / programmed death-ligand 1 (PD-L1) axis have demonstrated efficacy in the treatment of a variety of cancers, including NSCLC. Pembrolizumab is a monoclonal antibody $(\mathrm{mAb})$ which targets and blocks PD-1, and it is indicated as a single agent in patients with advanced/metastatic NSCLC tumors expressing PD-L1 (tumor proportion score (TPS) $>1 \%$ ) (Paz-Ares et al., 2018; Reck et al., 2016; Amrane et al., 2020, Mok et al., 2019). More specifically, pembrolizumab is indicated in patients without EGFR or ALK genomic aberrations in stage III disease where patients cannot receive surgical resection or definitive chemoradiation (i.e., chemotherapy with concurrent radiotherapy), as well as in patients with EGFR or ALK mutations that have continued disease progression even with FDA-approved

${ }^{1}$ Indiana University School of Medicine-Fort Wayne, 2101 E Coliseum Blvd E, Fort Wayne, IN 46805, USA. ${ }^{2}$ Medical Oncology, Parkview Cancer Institute, 11050 Parkview Circle, Fort Wayne, IN 46845, USA. *For Correspondence: takefumi.komiya@parkview.com 
therapy. Several randomized clinical trials support the use of pembrolizumab for locally advanced or metastatic, PD-L1-expressing NSCLC as it has been shown to help increase overall survival, PFS, and response compared to chemotherapy alone (Paz-Ares et al., 2018; Reck et al., 2016; Amrane et al., 2020, Mok et al., 2019).

This study is a retrospective case series of adult $(>18$ years old) patients with stage III NSCLC diagnosed at Parkview Health from 2019-2021 who were treated with pembrolizumab plus radiotherapy. We hypothesize that immunotherapy and radiation without chemotherapy may provide good disease control and tolerability in patients with unresectable stage III NSCLC that cannot be treated with chemoradiation.

\section{Materials and Methods}

\section{Study design}

This study was reviewed and approved by the Institutional Review Board at Parkview Health. This study is a retrospective chart review and analysis of the electronic medical records of adult patients diagnosed with stage III NSCLC at Parkview Health from 2019-2021 and treated with pembrolizumab as initial treatment plus sequential consolidation chest radiation (CXRT). As there is no prospective study design, this study is considered a case series. Eligible cases were screened by the Data Quality Management department at Parkview Cancer Institute and provided by the Principal Investigator. Patients who underwent surgery for a primary tumor and patients less than 18 years of age were excluded from the study. Study data were collected and managed through the Research Electronic Data Capture (REDCap) tool hosted at Parkview Health.

Patient demographics included year of birth, gender, and race. Diagnostic data included patient age at diagnosis, tumor pathology/cell type from biopsy, PD-L1 TPS score, primary tumor location, and clinical TNM stage according to the American Joint Committee on Cancer (AJCC) 8th edition staging system. Eastern Cooperative Oncology Group performance status (ECOG PS) scores, smoking history, and reasons for discontinuation were also noted.

Treatment data for pembrolizumab included total cycles administered, dose (mg), and frequency of dosing (3 weeks, 6 weeks, or other). Treatment data for CXRT included total grays delivered, fractions, and dose per fraction.

\section{Data analysis}

The primary endpoint of this study was response (tumor reduction). For response analysis, tumors were re-staged via computed tomography (CT) scan by noting the longest diameter (LD) of primary lesions and short axis of any pathologically enlarged hilar/mediastinal lymph nodes if present at baseline at various timepoints throughout therapy. These measurements were analyzed via the Response Evaluation Criteria in Solid Tumors version 1.1 (RECIST 1.1) to determine percent reduction in tumor size, presence/absence of new lesions, and how well patients responded to therapy.

\section{Results}

Patient sample characteristics

A total of 5 patients met the eligibility criteria of this study. There were 2 white males and 3 white females with a median age of diagnosis of 69 (range 62-84). All patients had an ECOG PS score of 2. For systemic treatment, all patients received $200 \mathrm{mg}$ cycles of pembrolizumab every 3 weeks until completion or discontinuation, and sequential CXRT was given after several doses of pembrolizumab. More detailed information can be found in Table 1. Primary tumor locations included 3 right-upper lobe (RUL) and 2 left-upper lobe (LUL). All tumors were characterized as having a PD-L1 TPS $>20 \%$ (median 80 , range 20-90). There were four cases of stage IIIA squamous cell carcinoma (SCC) and one case of stage IIIC adenocarcinoma. The four patients with stage IIIA SCC responded well to treatment thus far; specifically, two of these patients achieved partial response (PR) (38.9\% and $46.7 \%$ reduction in target lesion size, PD-L1 TPS 80\% and $90 \%$, respectively), one achieved complete response (CR) $(100 \%$ reduction in target lesion size, PD-L1 TPS $80 \%)$, and one remained at stable disease (SD) $(18.4 \%$ reduction in target lesion size, PD-L1 TPS 20\%). Despite two discontinuations of pembrolizumab in this group, one due to diarrhea and another due to pneumonitis, all four patients have continued without progression/relapse after receiving combination therapy. The patient with poorly differentiated stage IIIC adenocarcinoma developed metastasis to the brain after 4 cycles of pembrolizumab and subsequently died of disease.

\section{Discussion}

Landscape in the treatment of stage III NSCLC has changed over the last few years with the introduction of maintenance immunotherapy. Durvalumab is also a mAb that targets the PD-1 / PD-L1 axis and is indicated for unresectable stage III NSCLC without disease progression following concurrent platinum-based chemotherapy and radiation therapy (Antonia et al., 2017). The landmark "PACIFIC" study compared durvalumab as consolidation immunotherapy with placebo in patients with stage III, locally advanced, unresectable NSCLC who had no disease progression following $>2$ cycles of platinum-based chemoradiotherapy. Patients were randomly assigned in a $2: 1$ ratio to durvalumab $(10 \mathrm{mg} / \mathrm{kg}$ body weight, intravenous) and placebo groups, respectively, and received consolidation therapy every 2 weeks (Antonia et al., 2017). Durvalumab had greater median PFS (16.8 months vs. 5.6 months; HR, 0.52; 95\% CI, 0.42$0.65 ; \mathrm{P}<0.001), 12$-month PFS rate (55.9\% vs. $35.3 \%)$, 18 -month PFS rate ( $44.2 \%$ vs. $27.0 \%$ ), objective response rate $(28.4 \%$ vs. $16.0 \% ; \mathrm{P}<0.001)$, median duration of response $(72.8 \%$ vs. $46.8 \%$ ongoing response at 18 months), and median time to death/distant metastasis (23.2 months vs. 14.6 months; $\mathrm{P}<0.001)$ compared to placebo. In terms of safety, both adverse events of any grade and cause (96.8\% vs. $94.9 \%)$ and grade $3-4$ adverse events $(29.9 \%$ and $26.1 \%)$ were slightly higher in the durvalumab group, with the most common grade 
Table 1. Characteristics of Stage III NSCLC Cases

\begin{tabular}{|c|c|c|c|c|c|}
\hline Subject No. & 1 & 2 & 3 & 4 & 5 \\
\hline \multicolumn{6}{|l|}{ Demographics } \\
\hline Age at diagnosis (years) & 81 & 84 & 69 & 62 & 65 \\
\hline Sex & Male & Female & Female & Male & Female \\
\hline Race & White & White & White & White & White \\
\hline Histology & Squamous & Squamous & Squamous & Squamous & Adenocarcinoma \\
\hline PD-L1 TPS (\%) & 80 & 80 & 90 & 20 & 50 \\
\hline Primary tumor location & RUL & RUL & LUL & RUL & LUL \\
\hline Clinical stage & IIIA & IIIA & IIIA & IIIA & IIIC \\
\hline TNM stage (AJCC 8th) & $\mathrm{T} 2 \mathrm{aN} 2 \mathrm{M} 0$ & T4N0M0 & T4N0M0 & T3N1M0 & T4N3M0 \\
\hline ECOG performance status & 2 & 2 & 2 & 2 & 2 \\
\hline Smoking history & Former & Current & Current & Current & Former \\
\hline Reason for discontinuation & Pneumonitis & N/A & N/A & Diarrhea & Progression \\
\hline \multicolumn{6}{|l|}{ Pembrolizumab } \\
\hline Total cycles & 11 & 23 & $15+$ & 6 & 4 \\
\hline Cycles before CXRT & 7 & 7 & 4 & 5 & 4 \\
\hline Cycles after CXRT & 4 & 16 & $11+$ & 1 & 0 \\
\hline Dose (mg) & 200 & 200 & 200 & 200 & 200 \\
\hline Frequency of dosing & Every 3 weeks & Every 3 weeks & Every 3 weeks & Every 3 weeks & Every 3 weeks \\
\hline \multicolumn{6}{|l|}{ CXRT } \\
\hline Total grays (Gy) & 62 & 50 & 66 & 70 & N/A \\
\hline Fractions & 31 & 20 & 33 & 35 & N/A \\
\hline Dose per fraction & 2 & 2.5 & 2 & 2 & $\mathrm{~N} / \mathrm{A}$ \\
\hline Response (RECIST 1.1) (\%) & PR $(38.9 \%)$ & $\mathrm{CR}(100 \%)$ & PR $(46.7 \%)$ & $\mathrm{SD}(18.4 \%)$ & $\mathrm{PD}$ \\
\hline
\end{tabular}

RUL, right-upper lobe; LUL, left-upper lobe; AJCC, American Joint Commission on Cancer; ECOG, Eastern Cooperative Oncology Group; N/A, not applicable; CXRT, chest radiation; RECIST, Response Evaluation Criteria in Solid Tumors; PR, partial response; CR, complete response; SD, stable disease; PD, progressive disease.

3-4 adverse event being pneumonia (4.4\% vs. $3.8 \%$ in durvalumab and control groups, respectively) (Antonia et al., 2017). Thus, PFS and secondary endpoints favor durvalumab post-chemoradiotherapy in stage III NSCLC compared to chemoradiotherapy alone, and safety profiles are comparable between the two methods of treatment.

This case series holds preliminary evidence that immunotherapy followed by sequential CXRT provides good disease control and adequate tolerability for patients with unresectable stage III NSCLC. In this cohort, patients with stage IIIA SCC did not have disease progression and benefited appreciably from this approach, with the patient with the lowest PD-L1 TPS of $20 \%$ achieving target lesion reduction classified as $\mathrm{SD}$ and the three patients with PD-L1 TPS of $>80 \%$ achieving either PR or CR. That all four patients with PR, CR, or SD have yet to show any signs of progression is encouraging. Although the patient with poorly differentiated adenocarcinoma and PD-L1 TPS of $50 \%$ was noted to have primary lung lesion reduction with 4 cycles of pembrolizumab, she developed PD with brain metastasis, and the benefits/drawbacks of combination therapy should be investigated further and compared between the three NSCLC tumor histologies (i.e., adenocarcinoma, SCC, large cell carcinoma). Overall, this cohort of patients appeared to respond well to combination immunoradiotherapy as hypothesized, however larger studies lacking the limitations described below would be necessary to draw definitive conclusions.

Despite the known survival benefits of concurrent chemoradiation, combination chemotherapy, and introduction of maintenance durvalumab in unresectable stage III NSCLC, the severe toxicity associated with the chemotherapy aspect - particularly bone marrow suppression and reduction in health-related quality of life - removes a potential treatment option from patients with poor PS (Fujio et al., 2019), necessitating further evaluation of alternatives such as immunoradiotherapy. In general, the safety profile of immunotherapy is much more manageable than that of chemotherapy due to its specific and targeted nature.

Pembrolizumab immunotherapy plus radiotherapy, which was presented here as a case series, may be a good alternative to concurrent chemoradiation. This study is timely and important as there are currently no other studies in the literature looking at immunotherapy and radiotherapy without chemotherapy in locally advanced NSCLC, especially in the setting of poor ECOG PS.

\section{Limitations}

First, the small sample size and the lack of a control group prevent us from drawing clinically relevant conclusions regarding the benefits of immunoradiotherapy vs. immunotherapy alone in this patient population. A control group would have allowed further comparison 
via chi-squared/Fisher's exact test. Second, there was slight variation in the frequency of pembrolizumab administration due to patient preference and circumstance. Third, due to the recent timeframe of initial diagnosis (2019-2021), survival analysis could not be compared to the figures in the literature on 5-year overall survival, and a more longitudinal analysis should be conducted.

In conclusion, this retrospective case series suggests that combination immunoradiotherapy consisting of initial pembrolizumab treatment and sequential CXRT may be beneficial for stage III NSCLC patients with high PD-L1 expression, particularly in SCC, but further validation studies are needed.

\section{Author Contribution Statement}

All authors contributed to data collection, analysis, and preparation of manuscript.

\section{Acknowledgements}

We thank the Parkview Research Center for administrative support.

\section{Ethical approval and consent to participate}

IRB exempt status was given by Parkview health.

\section{Conflicts of interest}

TK and Parkview Health received a travel fund and research support from Merck, respectively. The other author declared that there are no conflicts of interest.

\section{References}

American Cancer Society. Key Statistics for Lung Cancer. Available from: https://www.cancer.org/cancer/lung-cancer/ about/key-statistics.html. [Last accessed on 3 Aug 2021].

Amrane K, Geier M, Corre R, et al (2020). First-line pembrolizumab for non-small cell lung cancer patients with PD-L1 $>50 \%$ in a multicenter real-life cohort: The PEMBREIZH study. Cancer Med, 9, 2309-16.

Antonia SJ, Villegas A, Daniel D, et al (2017). Durvalumab after chemoradiotherapy in stage III non-small-cell lung cancer. N Engl J Med, 377, 1919-29.

Aupérin A, Péchoux CL, Walter ER, et al (2010). Meta-analysis of concomitant versus sequential radiochemotherapy in locally advanced non-small-cell lung cancer. J Clin Oncol, 28, 2181-90.

Duma N, Santana-Davila R, Molina JR (2019). Non-small cell lung cancer: Epidemiology, Screening, Diagnosis, and Treatment. Mayo Clin Proc, 94, 1623-40.

Fujio T, Nakashima K, Naito T, et al (2019). Platinum combination chemotherapy is poorly tolerated in malnourished advanced lung cancer patients with poor performance status. Nutr Cancer, 71, 767-71.

Mok TSK, Wu Y, Kudaba I, et al (2019). Pembrolizumab versus chemotherapy for previously untreated, PD-L1-expressing, locally advanced or metastatic non-small-cell lung cancer (KEYNOTE-042): a randomised, open-label, controlled, phase 3 trial. Lancet, 393, 1819-30.

Mörth C, Valachis A (2014). Single-agent versus combination chemotherapy as first-line treatment for patients with advanced non-small cell lung cancer and performance status
2: a literature-based meta-analysis of randomized studies. Lung Cancer, 84, 209-14.

Paz-Ares L, Luft A, Vicente D, et al (2018). Pembrolizumab plus chemotherapy for squamous non-small-cell lung cancer. $N$ Engl J Med, 379, 2040-51.

Reck M, Rodríguez-Abreu D, Robinson AG, et al (2016). pembrolizumab versus chemotherapy for PD-L1-positive non-small-cell lung cancer. $N$ Engl J Med, 375, 1823-33.

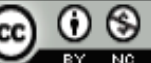

This work is licensed under a Creative Commons AttributionNon Commercial 4.0 International License. 